\title{
Non-intrusive In-flight Propeller Blade Deformation Measurements by Means of a Rotating Camera
}

\author{
Fritz Boden, Boleslaw Stasicki \\ German Aerospace Center DLR, Bunsenstraße 10, Göttingen, Germany \\ Email: fritz.boden@dlr.de
}

\begin{abstract}
:
The Image Pattern Correlation Technique (IPCT) is a powerful optical tool to measure the surface shape and deformation of aircraft structures, e.g. wings, propeller or rotor blades, non-intrusively in flight. The latest development of this technique is its application to $360^{\circ}$ propeller deformation measurements. Therefore a special stereo camera system rotating with the propeller at its full rotational speed was designed, whereby the system was exposed to extreme vibration and centrifugal forces. By beginning of 2014 the whole system had been successfully tested on an Evektor VUT 100 COBRA airplane. In the paper, this highly sophisticated rotary camera system and the measurement technique IPCT is described. Furthermore some results of the first flight tests are presented.
\end{abstract}

Key words: IPCT, flight testing, Propeller, AIM², deformation, rotating camera, optical method

\section{Motivation}

The knowledge about the behavior of propeller as well as rotor blades during operation is of high interest, because their shape and especially their local angle of attack directly influence the performance of the propeller or rotor.

The standard method to get this information is the measurement of the strain and acceleration at certain single locations on the blade by using strain gauges and accelerometers. By means of this data numerical models of the blade are validated giving an idea of the behavior during flight.

As shown in the past by several ground and flight test studies [1-4] the image pattern correlation technique (IPCT) is a very suitable tool to measure the shape and deformation of aircraft structures non-intrusively. Its applicability was still tested for in-flight propeller deformation measurements on the Piaggio P.180 airplane [4] by having a camera system installed in the luggage compartment of the airplane looking stroboscopically to the passing propeller blade. However, due to the limited optical access only a small part of the propeller revolution has been observed. Therefore the idea was born to design an imaging system rotating with the propeller and thus enabling the observation of the complete $360^{\circ}$ revolution of the blade.

In what follows the imaging system, the applied deformation measurement technique IPCT, as well as the performed test on the EVEKTOR VUT100 COBRA airplane are presented briefly.

\section{The IPCT method}

The IPCT is an optical deformation measurement which uses images of the observed object to determine its shape and location [5]. As shown in Fig. 1 the method requires images of the object taken from at least two cameras in a stereoscopic arrangement to obtain the $3 \mathrm{D}$ shape and location of the surface. Since the IPCT processing is based on digital image correlation (DIC) the surface has to be equipped with a stochastic dot pattern. For a proper processing, the locations as well as the optical properties of the cameras have to be known. This so called camera calibration can easily be done by placing a plate with a checker board of known size in the cameras field of view and recording it in different orientations.

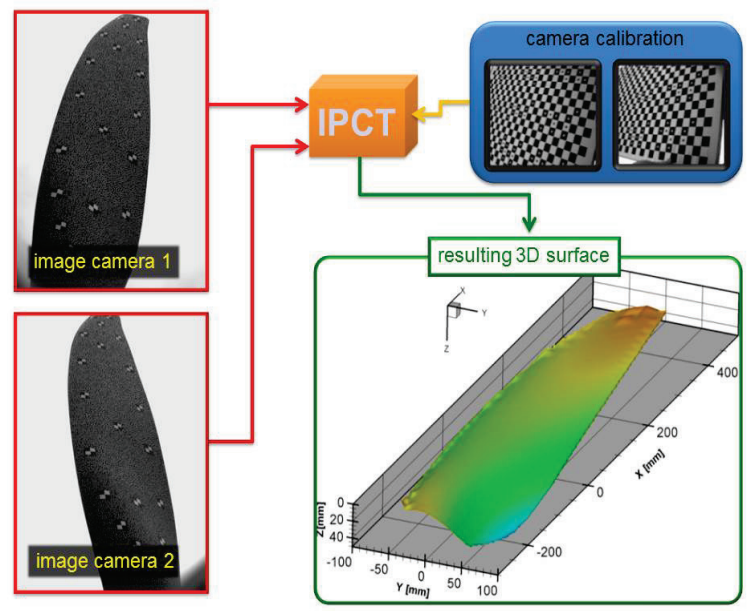

Fig. 1: Working principle of IPCT (required input and resulting output) 
In order to measure deformations recordings of the object in a reference state (e.g. propeller at standstill) and in a loaded state (e.g. propeller during flight manoeuvers) are processed by means of IPCT and the resulting surfaces are compared to each other.

In addition to the dot pattern checker board markers are applied to the surface. Those markers are used to get a first idea about the surface location and movement and for the recalibration of the cameras in case of any misalignment due to vibrations or deformations of the camera support. Furthermore some of the markers are able to be used as so called reference markers enabling the separation of solid body movements and real deformations.

The measurement accuracy of IPCT is in the order of 0.1 to 0.2 pixels on the camera sensor and thus normally in a range of $0.01 \%$ of the observed area. Depending on the applied optics, the geometric arrangement of the setup and the viewing conditions the accuracy can be tune in the range of some millimeters down to sub-millimeters. Fig. 2 shows the estimation of the inaccuracy of a setup similar to the later presented measurement setup for the propeller measurements according to the formulas given in [6]. The estimation is done for two different focal lengths $f(12.5 \mathrm{~mm}$ and $16 \mathrm{~mm})$ and a base distance of around $200 \mathrm{~mm}$. The inaccuracy along the line of sight is $\mathrm{dz}$, whilst the ones in perpendicular to the line of sight are $\mathrm{dx}$ and $\mathrm{dy}$. As the measurement of depth is less accurate (i.e. $d z$ ) the measurement setup is made in a kind that $\mathrm{dx}$ and $\mathrm{dy}$ are in the interesting directions of the measurement (e.g. for bending and twisting of the propeller blade). Compared to locally strain gauge measurements, the IPCT is less accurate, but it directly delivers 3D data of the complete measured surface.
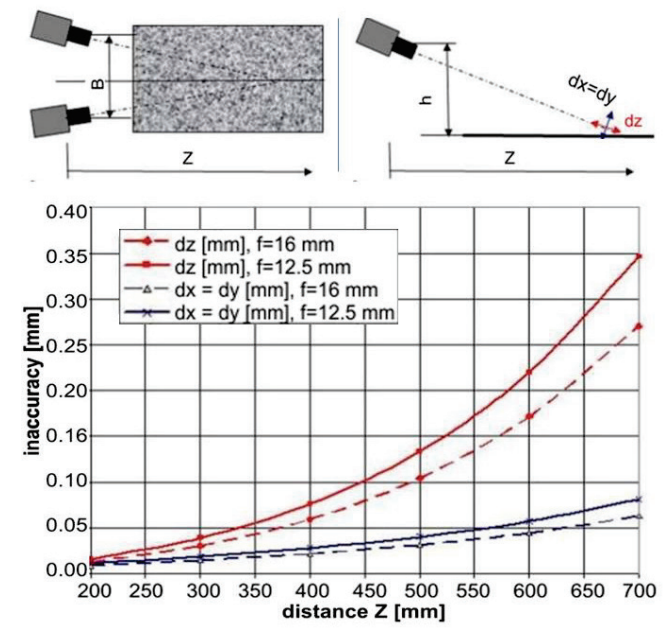

Fig. 2: Estimation of the inaccuracy of the presented measurement setup

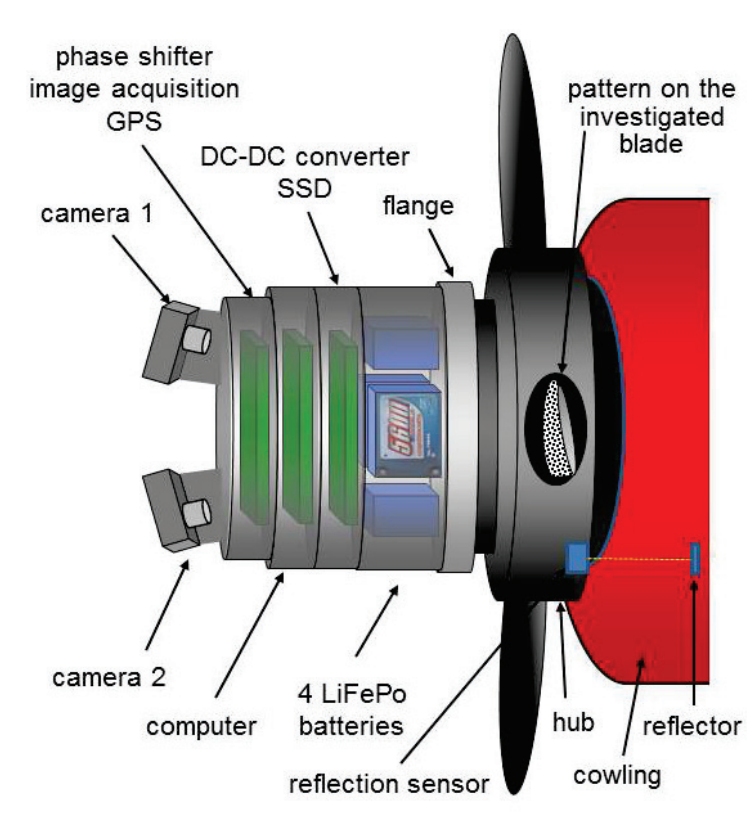

Fig. 3: Sketch of the rotating camera system

\section{Design of the rotating camera}

For applying the IPCT the recording of stereoscopic image pairs of the propeller blade and thus a camera system suitable for $360^{\circ}$ measurements are required. Even if it's the more difficult way to install a camera system it was decided to design a new imaging system to be mounted on the propeller hub co-rotating with the propeller.

A sketch of the designed rotating camera is shown in Fig. 3 and its simplified block circuit in Fig. 4.

The system consists of a double CMOS sensor stereo camera system, a digital phase shifter, an image acquisition, a reflection sensor to get one trigger pulse per revolution and an embedded computer system with WLAN and GPS. All components are powered by four rechargeable LiFePO batteries with a total voltage of $14.8 \mathrm{~V}$ and a capacity of $3.5 \mathrm{Ah}$ which is sufficient for 80 minutes of continuous image recording with a maximum speed of 45 image pairs per second.

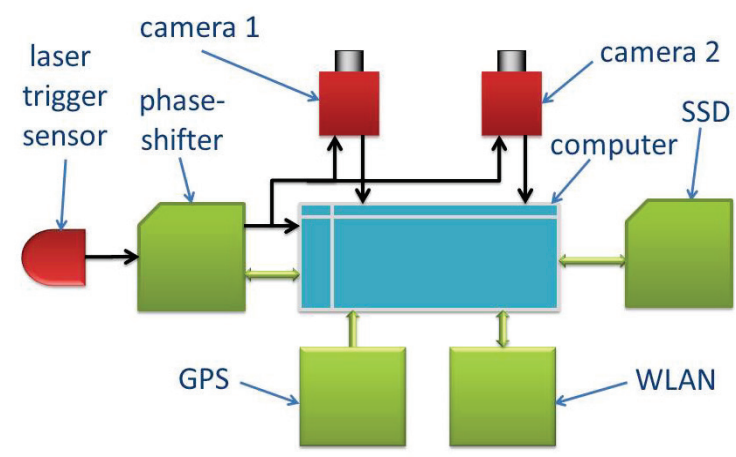

Fig. 4: Simplified block circuit of the camera system 


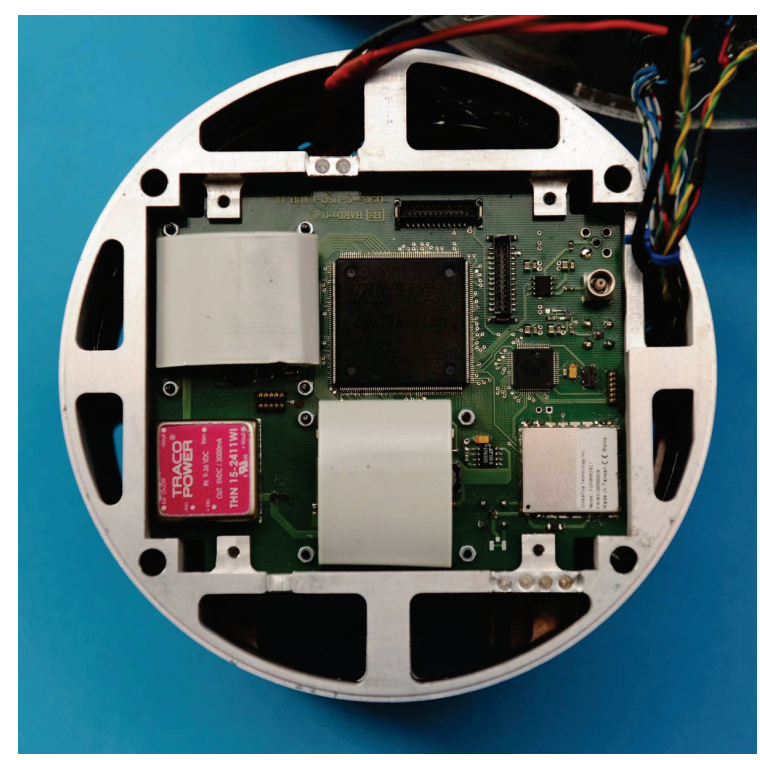

Fig. 5: An electronic board fixed within the rigid frame

All electronic boards and other system components are fixed in a rigid metal frames preventing their damage due to the centrifugal forces and vibration. The principle of this construction is displayed in Fig. 5. These metal frames are stacked one upon the other and linked by four rods forming the complete system cabinet as shown in Fig. 6.

To get a suitable stereoscopic viewing angle to the same field of view on the propeller blade the two cameras (camera 1 and camera 2) are located as far away as possible from the propeller with a maximum base value i.e. the distance between the both cameras.

\section{Principle of operation}

By means of the laser type reflection sensor aimed at a reflector on the cowling, one trigger pulse is created for each revolution of the propeller. Depending on the setting of the phase shifter a stereoscopic image pair is recorded directly at the same instant of time as the trigger pulse or with a specific delay (phase shift). This phase shift can either be set to a constant value for recording the propeller at the same position for each revolution, independent of the propeller speed, or with a phase shift change for each revolution by a given increment, hence providing scans of a phase angle range i.e. also for a complete $360^{\circ}$ revolution. The principle of this phase shifting has been patented in several countries [7]. The images taken by the CMOS sensors are transmitted to the embedded miniature computer via two GigE interfaces and stored on an integrated SSD in real-time together with the present GPS data like time and location.

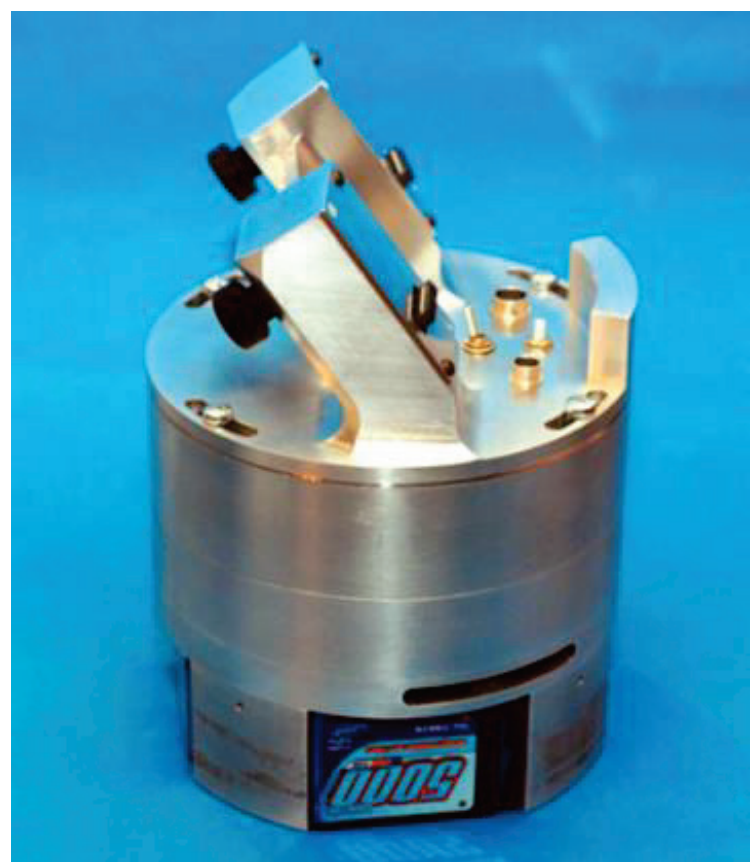

Fig. 6: The complete camera system (without adapter)

By means of the WLAN connection the recorded images can be viewed and examined directly in the cabin during the flight test. If required the image acquisition parameters can be instantly modified.

After the test the batteries and the SSD with the acquired images can be easily replaced and the next flight test can be started after a very short time. The data stored on the SSD can be processed with the IPCT software on a postprocessing computer.

\section{Digital mock-up}

In order to test the viewing conditions of the cameras in advance and thus defining the focal length of the lenses the complete setup was simulated by means of a digital mock-up (DMU). The DMU included the 3D model of the rotating camera system, a 3D laser scan of the propeller hub and the blade and the simulated optics and camera sensor. Fig. 7 shows a screenshot of the DMU, that is based on Catia V5 and several macro scripts [5]. By adding cameras with the same optical properties like for the later setup to a Catia 3D scenery the images seen by those cameras later can be simulated. In addition the dot pattern and the markers required for the IPCT measurements can optimally be designed because the pattern is applied to the CAD surfaces as a "sticker".

To ensure an optimal size and shape of the markers and the dots the pattern is designed in a progressive way. 


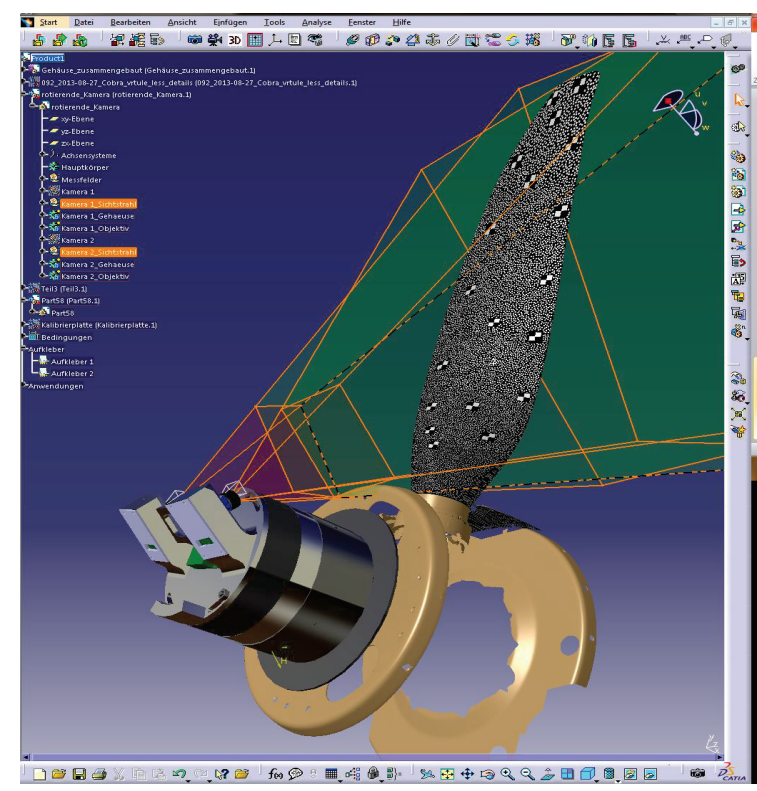

Fig. 7: Screenshot of the digital mock-up (DMU)

That means, the dots and the markers become bigger towards the blade tip and are stretched in spanwise direction. In Fig. 8 the final designed pattern for the propeller measurements is shown and in Fig. 9 the camera images simulated in the DMU can be seen. It is clearly visible, that the progressive design of the pattern yields to the impression of all markers and dots having the same size within the camera images.

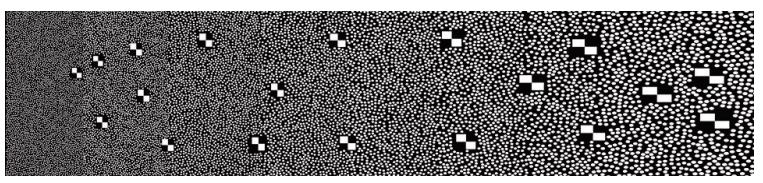

Fig. 8: Pattern designed for the blade

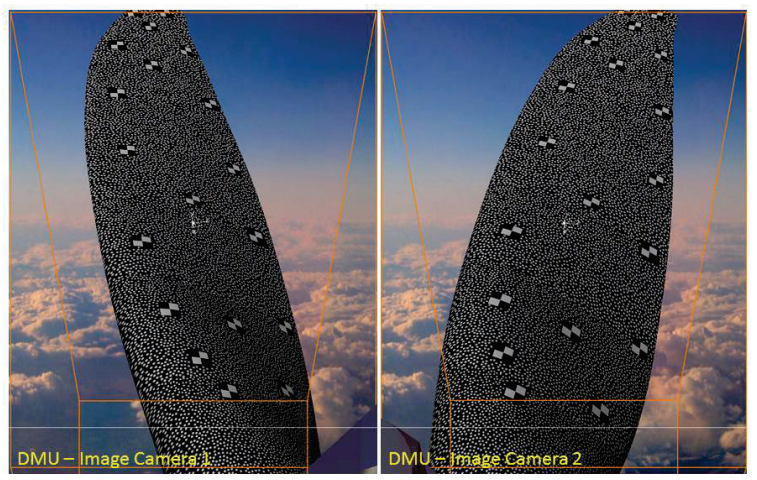

Fig. 9: Example of camera images simulated by means of the DMU

\section{Flight test preparation}

After the completion of the rotating camera prototype for flight test and the design of the pattern, the hardware has been transported to AVIA Propeller in Prague. There one of the blades was painted with the required dot pattern.
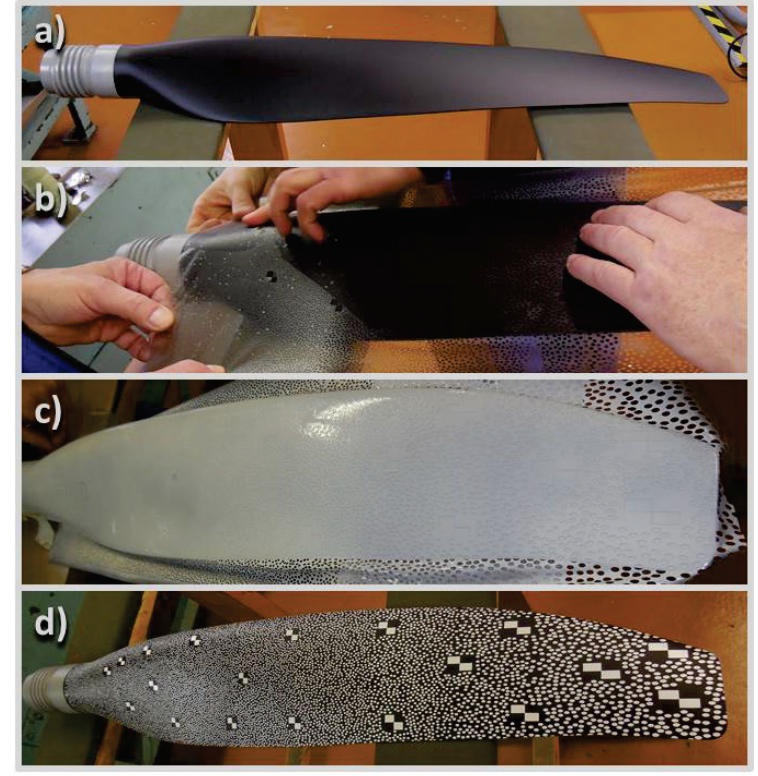

Fig. 10: Painting of the blade (a - black primed blade, $b$ - application of the paint mask, $c$ - application of white paint, $d$ - final painted blade)

To perform this in a proper way, a paint mask was created from the designed pattern file. The paint mask was applied to the black primed blade and white color was sprayed onto the blade (Fig. 10). Finally the mounting of the camera to the propeller and the static balancing of the complete system took place. Following the propeller and the camera were transported to the EVEKTOR site in Kunovice (CZ) and mounted to the VUT-100 COBRA experimental airplane. For checking the function and setting the camera focus as well as other basic testing with the propeller at standstill, the rotating camera has a connector for keyboard, mouse and monitor as well as a ground power plug. Fig. 11 shows the measurement setup during final ground preparation.

\section{Flight testing}

Following to the ground preparation of the measurement setup as mentioned above, the camera system was calibrated by placing a flat plate with a checker board pattern in its field of view (see Fig. 12).

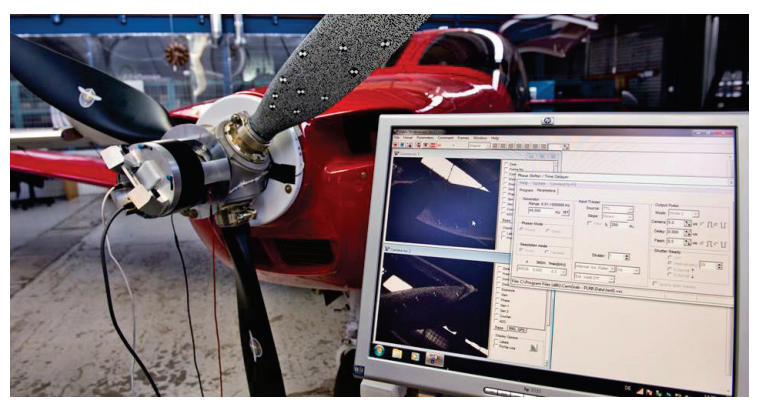

Fig. 11: Rotating camera mounted to the experimental aircraft, monitor connected for final adjustment 


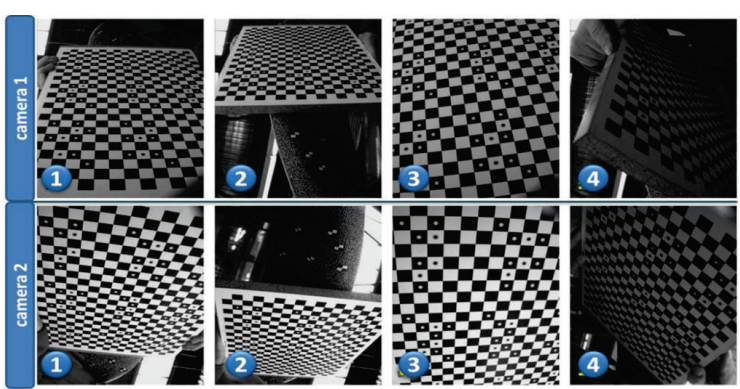

Fig. 12: Example images of the two cameras recorded during calibration

Like for the later flight test, the rotating camera system for this purpose was powered by the integrated batteries and controlled via WLAN connection and remote desktop. Since the WLAN connection between the camera and the flight test engineers notebook in the airplane was not stable enough for controlling the camera in-flight all camera parameters were set and the recording was started before starting the engine and performing the flight test. Thus the camera acquired images for the complete flight trial from engine start to engine stop.

In total four flights have been performed including different propeller settings, propeller loadings and flight manoeuvers. Several problems concerning triggering, unstable camera connection and difficult illumination conditions were solved during those flight activities. Altogether around 40,000 image pairs were recorded. Some example images are shown in Fig. 13.

\section{Image Data Processing and Results}

After the test, the SSD with the recorded images was taken out of the camera and the data was copied to a PC with the IPCT processing software, developed by DLR.

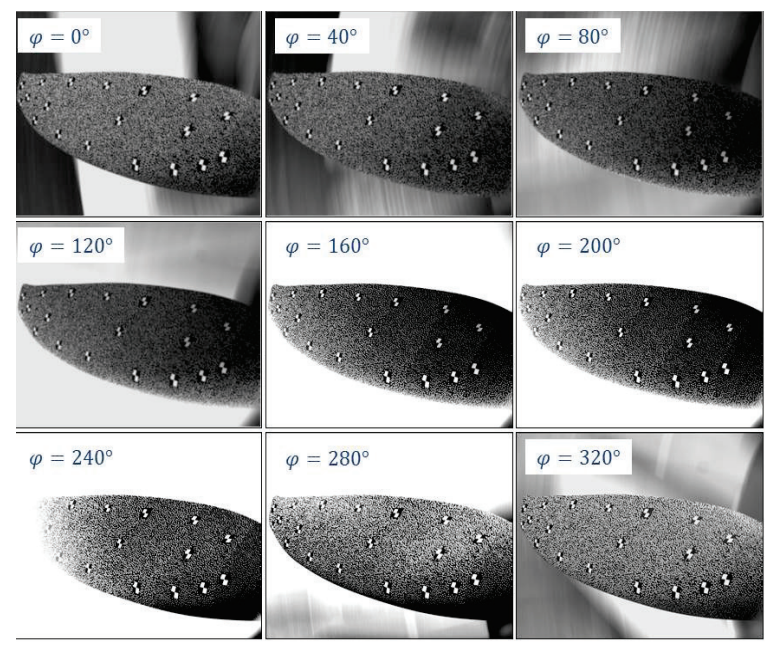

Fig. 13: Example images of one camera during rotation ( $\varphi$ is the propeller phase angle)

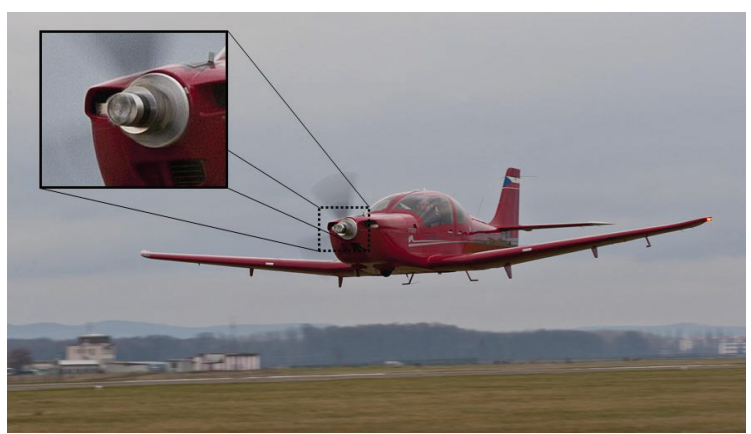

Fig. 14: EVEKTOR VUT-100 Cobra flying with rotating camera mounted on its propeller

The tool uses the calibration images to obtain all relevant parameters of the camera system and the transformation matrix between camera coordinates and 3D world coordinates. In the next step, the measurement image pairs are evaluated, first by detecting the checker board markers on the blade, second by finding corresponding pattern regions in camera 1 and camera 2. The final result of the IPCT processing (a detailed description can be found in [5]) is the 3D surface of the measured propeller blade during the instant of time of the recording. Fig. 15 shows two example surfaces resulting from the reference recording of the propeller blade at standstill and one during flight. The reference surface is plotted transparent whilst the in-flight surface is plotted solid with the $\mathrm{Z}$ coordinate as color code for a better visibility of the 3D shape. The spanwise direction of the blade is in line with the $X$ coordinate (blade tip at $X$ around $400 \mathrm{~mm}$ ). The chord-wise direction of the blade is in line with the $Y$ coordinate (leading edge $-Y$, trailing edge $+Y)$. The measured shape of the blade is in good agreement with the real blade. As expected under load, the blade shows an increasing bending towards the blade tip. If a line of surface points is extracted along the Xdirection, the bending of the blade can directly be read out of the diagram as shown in Fig. 16.

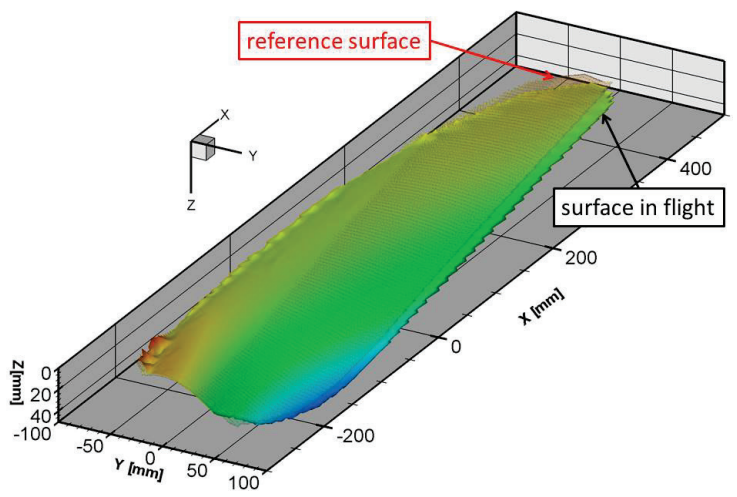

Fig. 15: Example resulting surfaces (transparent reference surface of the propeller blade at standstill, solid - surface of the blade in flight (color code corresponds qualitatively to the Z-coordinate)) 


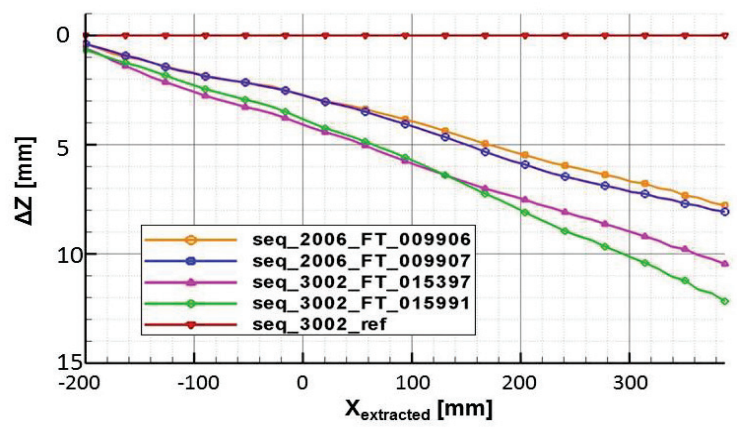

Fig. 16: Bending of the blade (points extracted for different measurement points along the $X$-coordinate and normalized to the reference recording)

The given value of $\Delta Z$ is the difference of the measured $Z$ coordinate during flight compared to the reference surface. The maximum deflection occurred during the test is about $12 \mathrm{~mm}$ at the blade tip.The extraction of points can also be performed along the $\mathrm{Y}$-coordinate as shown in Fig. 17. Thus the local blade pitch angle compared to the reference surface can be shown easily.

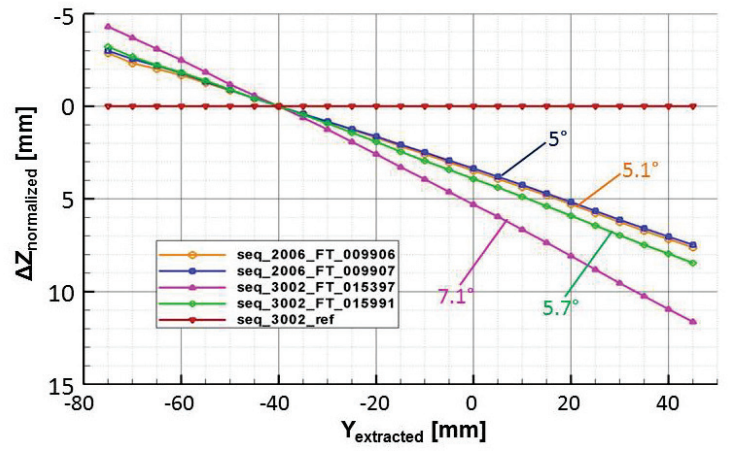

Fig. 17: Visualization of the change of the pitch angle (points extracted for different measurement points along the $Y$-coordinate and normalized to the reference surface)

\section{Conclusions}

A rotating and self-contained stereoscopic camera system for airplane propeller deformation measurements was designed, built and successfully tested on an EVEKTOR VUT100 COBRA airplane. This small aircraft has been chosen as a "worst case test" because of the largest vibration and highest propeller rpm. To measure the shape of the observed propeller blade and thus its real local pitch angle and deformation, the recorded image pairs have been processed by the optical deformation measurement technique IPCT. The installation effort on the airplane was very low. Only the pattern had to be applied and the camera had to be mounted. A classical installation of strain gauges for a similar surface measurement would have been nearly impossible due to the required number of sensors and the fitting on the propeller would have taken much longer. After the measurement the camera and the dot pattern could be removed easily and the airplane used as before. By using another flange, the rotating camera can also be applied to measurements on other airplanes and with some modification also on large aircraft propellers. With the lessons learned on the small airplane similar devices can now be designed to carry out measurements on larger aircrafts, rotors of helicopters or wind turbines. For this first test, the camera recorded one image pair per revolution, yielding to a frame rate of around $45 \mathrm{~Hz}$. Thus only the lowest blade oscillations are measureable with this camera. With future camera sensors with higher frame rate also measurements of blade modes with higher frequency will be possible.

\section{References}

[1] F. Boden, T. Kirmse, T. Weikert, T. Wolf, C. Petit, H.W. Jentink: Application of a new optical measurement technique for non-intrusive wing deformation measurements on a large transport aircraft, $21^{\text {st }}$ SFTE (EC) Symposium, Vergiate, Agusta Westland, paper 4, $3^{\text {rd }}-6^{\text {th }}$ October 2010

[2] H.P.J. Veerman, H. Kannemans, H.W. Jentink: High Accuracy In-Flight Wing Deformation Measurements based on Optical Correlation Technique, NLR-TP-2008-718, $19^{\text {th }}$ SFTE (EC) Symposium, Manching, EADS, Germany, paper $3-2,22^{\text {nd }}-24^{\text {th }}$ September 2008

[3] F. Boden, C. Maucher: Blade Deformation Measurements with IPCT on an EC 135 Helicopter Rotor, in: Research Topics in Aerospace Advanced In-Flight Measurement Techniques (Chapter 13), Springer Verlag, Heidelberg New York Dordrecht London, 2013, Pages 195-213. ISBN 978-3-642-34737-5 . ISSN 2194-8240

[4] C. Lanari, B. Stasicki, F. Boden, A. Torres: Image Based Propeller Deformation Measurements on the Piaggio P180, in: Research Topics in Aerospace Advanced In-Flight Measurement Techniques (Chapter 9). Springer Verlag, Heidelberg New York Dordrecht London, 2013, Pages 133-153. ISBN 978-3-642-34737-5 . ISSN 2194-8240

[5] F. Boden, T. Kirmse, H. Jentink: Image Pattern Correlation Technique (IPCT)" in: AIM ${ }^{2}$ Advanced Flight Testing Workshop - HANDBOOK of ADVANCED IN-FLIGHT MEASUREMENT TECHNIQUES BoD - Books on Demand, Norderstedt, 2013, pages 63 to 85. ISBN 978-37322-3740-1, 2013

[6] K. KRAUS: Photogrammetry: geometry from images and laser scans, $22^{\text {nd }}$ edition, Walter DeGruyter, Berlin, 2007.

[7] Digitaler Phasenschieber, DLR-Patent DE 19544 642 